\title{
Characteristics and outcomes of culture-negative versus culture-positive severe sepsis
}

\author{
Jason Phua ${ }^{1,2^{*}}$, Wang Jee Ngerng ${ }^{1,2}$, Kay Choong See ${ }^{1,2}$, Chee Kiang Tay ${ }^{1,2}$, Timothy Kiong ${ }^{3}$, Hui Fang Lim ${ }^{1,2}$, \\ Mei Ying Chew ${ }^{1,2}$, Hwee Seng Yip ${ }^{1,2}$, Adeline Tan ${ }^{4}$, Haji Jamil Khalizah ${ }^{5}$, Rolando Capistrano ${ }^{1,2}$, Kang Hoe Lee ${ }^{6}$ and \\ Amartya Mukhopadhyay ${ }^{1,2}$
}

\begin{abstract}
Introduction: Culture-negative sepsis is a common but relatively understudied condition. The aim of this study was to compare the characteristics and outcomes of culture-negative versus culture-positive severe sepsis.

Methods: This was a prospective observational cohort study of 1001 patients who were admitted to the medical intensive care unit (ICU) of a university hospital from 2004 to 2009 with severe sepsis. Patients with documented fungal, viral, and parasitic infections were excluded.

Results: There were 415 culture-negative patients (41.5\%) and 586 culture-positive patients (58.5\%). Gram-positive bacteria were isolated in 257 patients, and gram-negative bacteria in 390 patients. Culture-negative patients were more often women and had fewer comorbidities, less tachycardia, higher blood pressure, lower procalcitonin levels, lower Acute Physiology and Chronic Health Evaluation II (median 25.0 (interquartile range 19.0 to 32.0) versus 27.0 (21.0 to 33.0), $P=0.001$ ) and Sequential Organ Failure Assessment scores, less cardiovascular, central nervous system, and coagulation failures, and less need for vasoactive agents than culture-positive patients. The lungs were a more common site of infection, while urinary tract, soft tissue and skin infections, infective endocarditis and primary bacteremia were less common in culture-negative than in culture-positive patients. Culture-negative patients had a shorter duration of hospital stay (12 days (7.0 to 21.0) versus 15.0 (7.0 to27.0), $P=0.02$ ) and lower ICU mortality than culture-positive patients. Hospital mortality was lower in the culture-negative group (35.9\%) than in the culture-positive group (44.0\%, $P=0.01$ ), the culture-positive subgroup, which received early appropriate antibiotics $(41.9 \%, P=0.11)$, and the culture-positive subgroup, which did not $(55.5 \%, P<0.001)$. After adjusting for covariates, culture positivity was not independently associated with mortality on multivariable analysis.
\end{abstract}

Conclusions: Significant differences between culture-negative and culture-positive sepsis are identified, with the former group having fewer comorbidities, milder severity of illness, shorter hospitalizations, and lower mortality.

\section{Introduction}

Severe sepsis is a major cause of morbidity and mortality in both developed and developing countries [1]. Mortality rates remain high at $30 \%$ and rise to $60 \%$ in the presence of septic shock despite significant advancement in treatment modalities [2]. Bacteria are by far the most common causative microorganisms in sepsis, and cultures are positive in about $50 \%$ of cases [3]. Failure to administer antibiotics to which the pathogens are susceptible is

\footnotetext{
* Correspondence: jason_phua@nuhs.edu.sg

'Division of Respiratory and Critical Care Medicine, University Medicine Cluster, National University Hospital, National University Health System Tower Block Level 10, 1 E Kent Ridge Road, Singapore 119228

Full list of author information is available at the end of the article
}

associated with increased mortality [4]. Thus, early broad-spectrum antibacterial agents are recommended as a means to improve survival [5].

Less is known though about the other half of the equation: sepsis for which etiologic agents are not found. It is commonly thought that cultures may lack the sensitivity to detect all infecting bacteria [6]. Beyond this, and aside from data from a few multicenter epidemiological studies, which suggest that severity of illness and mortality are not significantly affected by microbiological documentation in sepsis [7-12], the medical literature is surprisingly devoid of information about patients with culture-negative sepsis.
C Biomed Central 
The aim of our study was hence to compare the characteristics and outcomes of culture-negative versus culturepositive severe sepsis.

\section{Materials and methods Study design}

This was a prospective observational cohort study conducted in the medical intensive care unit (ICU) of our university hospital. The study, being non-interventional, was approved by our institutional review board, the National Healthcare Group's Domain Specific Review Board, with a waiver of informed consent.

\section{Inclusion criteria}

We included all patients who were admitted to our ICU from 2004 to 2009 for severe sepsis, which was defined according to the 1992 American College of Chest Physicians (ACCP)/Society of Critical Care Medicine (SCCM) Consensus Conference criteria, that is, sepsis with at least one organ dysfunction [13]. The diagnosis of sepsis required the presence of the systemic inflammatory response syndrome due to infection.

\section{Exclusion criteria}

As we were interested in comparing acute culture-negative sepsis with culture-positive bacterial sepsis, we excluded patients with microbiogically proven fungal, viral, and parasitic infections, and tuberculosis. We only recorded the first ICU admission and excluded readmissions.

\section{Diagnosis of infection}

Infection was diagnosed clinically by the managing physicians. From the year 2005 onward, reference was made to the International Sepsis Forum Consensus Conference guidelines on definitions of infections where appropriate [14]. Briefly, the diagnosis of pneumonia required a radiographic infiltrate plus a high clinical suspicion, including fever or hypothermia, leukocytosis or leukopenia, and purulent respiratory secretions. Patients were deemed culture-positive if etiologic agents were recovered from blood or pleural fluid, or if semi-quantitative cultures of sputum, blind endotracheal aspirates, or bronchoalveolar lavage found moderate to heavy growths of bacteria with few epithelial cells seen on Gram stain examination ( $\leq 10$ per high power field). Intra-abdominal infections included but were not limited to intraabdominal abscesses, peritonitis, biliary tract infections, pancreatic infections, enteritis, and colitis. Urinary tract infection was diagnosed through typical signs and symptoms including fever, urgency, frequency, dysuria, pyuria, hematuria, positive Gram stain, pus, and suggestive imaging. Urine cultures were deemed positive with the isolation of $>10^{5}$ colony forming units $(\mathrm{cfu}) / \mathrm{mL}$ of microorganisms (or $10^{3} \mathrm{cfu} / \mathrm{mL}$ in catheterized patients).
Soft tissue and skin infections included surgical site infections, cellulitis, and necrotizing fasciitis. Infective endocarditis was diagnosed based on the revised Duke criteria. When diagnosing bacteremia, common skin contaminants like coagulase-negative staphylococci, Bacillus species, Corynebacterium species, micrococci, and Propionibacterium species were disregarded unless they were deemed clinically significant by the managing physicians or cultured from two or more blood cultures. Primary bacteremia was diagnosed when the microorganism cultured was not related to an infection at another site. Catheter-related sepsis is the only infection for which microbiological confirmation was mandated by the International Sepsis Forum [14]. For this study, the diagnosis of culture-positive catheter-related sepsis required a positive peripheral blood culture, while the diagnosis of culture-negative catheter-related sepsis was made clinically in the presence of pus or cellulitis at the exit site or tunnel tract infection.

\section{Clinical management}

Patient care in the ICU was left to the discretion of the managing physicians, who were encouraged to follow the Surviving Sepsis Campaign guidelines after they were published in 2004 [15]. While the treatments were not protocolized, broadly, they involved aggressive fluid resuscitation and vasopressors, with hemodynamic information obtained via lactate and $\mathrm{N}$-terminal B-type natriuretic peptide measurements, transthoracic echocardiography, arterial pressure waveform analyses, and transpulmonary thermodilution when indicated. Early intubation was advocated for respiratory failure. Blood cultures were obtained early, with $20 \mathrm{~mL}$ of blood distributed equally for each set of aerobic and anaerobic media, while cultures of other sites were performed depending on the source of infection. Empiric broad spectrum antibiotics were chosen based on the suspected infection and optimized and/or de-escalated according to the culture results.

\section{Data collection}

A research coordinator (WJN) prospectively entered the data into a Computerized Clinical Research Database under the close supervision of the principal investigator (JP). Patients were followed till discharge from or death in the hospital. The inputted data and electronic case records for all patients were then retrospectively reviewed by the co-investigators. Data including statistical outliers that might represent entry errors were verified and corrected in cases of inconsistency.

Data collected were baseline variables on entry to the ICU including patient demographics, source and time of admission, comorbidities, vital signs and blood investigations (white blood cell count, procalcitonin, and 
C-reactive protein where available), and variables on the first day of ICU admission including the Acute Physiology and Chronic Health Evaluation (APACHE) II and the corresponding Acute Physiology Score, the Sequential Organ Failure Assessment (SOFA) score, and treatment provided (vasoactive agents, mechanical ventilation, renal replacement therapy, and glucocorticoids for septic shock). We defined organ failures as a SOFA score of $>2$ for the organs concerned [11]. We documented the site(s) of infection based on the clinical impression of the managing physicians. To ensure that any bacteria isolated were the cause of severe sepsis that resulted in ICU admission, we recorded results of all bacteria cultures collected within the two days before and the two days after admission, unless they were deemed to be colonizers or contaminants by the managing physicians; in the latter cases, adjudication was provided by the principal investigator (JP). Bacteria isolated more than two days before ICU admission were only logged if they were judged to have led to the clinical deterioration by the managing physicians. We charted all antibiotics administered on the day of ICU admission and defined the initial antimicrobial therapy as appropriate if positive cultures were susceptible to any of these antibiotics or if all cultures were negative, and as inappropriate if positive cultures were not susceptible to all of these antibiotics [4].

The primary outcome variable was hospital mortality, while the secondary outcome variables were ICU mortality, duration of mechanical ventilation, ICU stay, and hospital stay.

\section{Statistical analyses}

We classified the patients into two groups depending on whether bacteria which caused the severe sepsis were found (culture-positive) or not found (culture-negative). We expressed categorical variables as number (percentage). After using the Kolmogorov-Smirnov test and examining histograms to verify if normality and homogeneity assumptions were satisfied, we expressed normally distributed numerical variables as mean (95\% confidence interval (CI)) and other numerical variables as median (interquartile range). We compared categorical variables using the $\chi^{2}$ test or Fisher exact test, normally distributed quantitative variables with the $t$ test, and other quantitative variables with the Mann-Whitney $U$ test. We used the Bonferroni correction for pairwise comparisons.

To identify the independent predictors of hospital mortality, in addition to univariable analyses, we performed a multivariable logistic regression analysis using a model that included variables which could potentially affect survival, that is, all recorded variables at baseline and on day one in the ICU, the site of infection, whether the patients were culture-negative or culture-positive, whether the initial antimicrobial therapy was appropriate or inappropriate, and whether bacteremia was absent or present. We looked for multicollinearity, and assessed model fit using the Hosmer-Lemeshow goodness-of-fit test. To identify the specific bacteria that were independently associated with mortality, we repeated the regression analysis after substituting the five commonest Gram-negative microorganisms and the five commonest Gram-positive microorganisms for the broad groups of culture negativity versus culture positivity as covariates into the model. We considered a $P$ value of $<0.05$ significant and used IBM SPSS version 20.0 (IBM Corp, Armonk, NY, USA).

\section{Results}

The study included 415 culture-negative patients (41.5\%) and 586 culture-positive patients (58.5\%) who were admitted to our ICU for severe sepsis. Table 1 describes their characteristics at baseline and on day one of the ICU stay. Compared to culture-positive patients, culturenegative patients were more likely to be women, have fewer comorbid conditions, less tachycardia, higher blood pressure, lower procalcitonin levels, lower APACHE II and SOFA scores, and less cardiovascular, central nervous system, and coagulation failures. Culture-negative patients were less likely to be treated with vasoactive agents on the first day of ICU stay.

As shown in Table 2, the lungs were commoner sites of infection, while liver abscesses, biliary tract, urinary tract, soft tissue and skin infections, infective endocarditis and primary bacteremia were less common in culture-negative than in culture-positive patients.

Table 3 lists the cultures performed within the two days before and the two days after ICU admission and the culture positivity rates. While more cultures were obtained from bile, liver abscesses, and soft tissue and skin in the culture-positive group than in the culturenegative group, there were no significant differences in the proportion of patients for which other cultures were performed in the two groups. Blood, urine, and endotracheal aspirate cultures were most frequently performed. A median of two blood cultures, one urine culture, and one endotracheal aspirate culture were obtained for both the culture-negative and the culture-positive patients.

Table 4 features the microbiology. Gram-positive bacteria were isolated in 257 patients $(25.7 \%)$ while Gramnegative bacteria were isolated in 390 patients (39.0\%). Among these patients, 196 (19.6\%) had only Gram-positive infections, 329 (32.9\%) only Gram-negative infections, while 61 (6.1\%) had mixed Gram-positive and Gramnegative infections. Staphylococcus aureus and Klebsiella pneumonia were the commonest Gram-positive and Gram-negative microorganisms respectively. 
Table 1 Characteristics at baseline and on day one of intensive care unit admission.

\begin{tabular}{|c|c|c|c|}
\hline & Culture-negative patients $(n=415)$ & Culture-positive patients $(n=586)$ & $P$ value \\
\hline \multicolumn{4}{|l|}{ Demographics } \\
\hline Age, years & $62.0(50.0-74.0)$ & $64.0(50.1-74.0)$ & 0.62 \\
\hline Men & $236(56.9)$ & $375(64.0)$ & 0.02 \\
\hline Race & & & 0.09 \\
\hline Chinese & $229(55.2)$ & $358(61.1)$ & \\
\hline Malay & $106(25.5)$ & $120(20.5)$ & \\
\hline Indian & $56(13.5)$ & $64(10.9)$ & \\
\hline Others & $24(5.8)$ & $44(7.5)$ & \\
\hline Source of admission & & & 0.49 \\
\hline Emergency department & $195(47.0)$ & $257(43.9)$ & \\
\hline Hospital floor & $195(47.0)$ & $293(50.0)$ & \\
\hline Other ICU & $25(6.0)$ & $34(5.8)$ & \\
\hline Operating room/recovery & $0(0)$ & $2(0.3)$ & \\
\hline \multicolumn{4}{|l|}{ Time of ICU admission } \\
\hline Day of hospital admission & $0(0-2.00)$ & $0(0-2.00)$ & 0.38 \\
\hline Office hour admission & $166(40.0)$ & $260(44.4)$ & 0.17 \\
\hline \multicolumn{4}{|l|}{ Comorbid conditions } \\
\hline Diabetes mellitus & $141(34.0)$ & $234(39.9)$ & 0.06 \\
\hline Chronic heart failure & $22(5.3)$ & $40(6.8)$ & 0.32 \\
\hline Chronic kidney disease & 48 (11.6) & $96(16.4)$ & 0.03 \\
\hline COPD & $24(5.8)$ & $38(6.5)$ & 0.65 \\
\hline Cancer & $29(7.0)$ & $55(9.4)$ & 0.18 \\
\hline Hematological malignancy & $9(2.2)$ & $24(4.1)$ & 0.09 \\
\hline No. of comorbid conditions ${ }^{a}$ & & & 0.004 \\
\hline 0 & $214(51.6)$ & $241(41.1)$ & \\
\hline 1 & $136(32.8)$ & $225(38.4)$ & \\
\hline 2 & $59(14.2)$ & $98(16.7)$ & \\
\hline$\geq 3$ & $6(1.4)$ & $22(3.8)$ & \\
\hline \multicolumn{4}{|l|}{ Vital signs upon ICU admission } \\
\hline Temperature, ${ }^{\circ} \mathrm{C}$ & $37.0(36.0-38.0)$ & $37(36.2-38.0)$ & 0.29 \\
\hline Heart rate, /min & $107.3 \pm 27.5$ & $113.4 \pm 25.9$ & $<0.001$ \\
\hline Mean blood pressure, $\mathrm{mm} \mathrm{Hg}$ & $78.0(64.0-96.0)$ & $75.0(62.0-91.0)$ & 0.01 \\
\hline Respiratory rate, /min & $24.0(20.0-31.0)$ & $25.0(20.0-31.0)$ & 0.84 \\
\hline \multicolumn{4}{|l|}{ Investigations $^{\mathrm{b}}$} \\
\hline White blood cell, $10^{9} / \mathrm{L}$ & $13.27(9.18-19.47)$ & $13.44(6.58-19.65)$ & 0.19 \\
\hline Procalcitonin, ng/mL & $3.95(0.90-17.28)$ & $16.40(2.74-47.03)$ & $<0.001$ \\
\hline C-reactive protein, mg/L & $47.50(14.05-191.50)$ & $51.50(11.63-181.50)$ & 0.53 \\
\hline \multicolumn{4}{|l|}{ Severity scores } \\
\hline APACHE ॥ & $25.0(19.0-32.0)$ & $27.0(21.0-33.0)$ & 0.001 \\
\hline Acute Physiology Score & $20.0(14.0-26.0)$ & $22.0(16.8-28.3)$ & $<0.001$ \\
\hline SOFA & $9.0(6.0-12.0)$ & $10.0(8.0-13.0)$ & $<0.001$ \\
\hline \multicolumn{4}{|l|}{ Organ failure } \\
\hline Renal & $88(21.2)$ & $127(21.7)$ & 0.86 \\
\hline Respiratory & $239(57.6)$ & $344(58.7)$ & 0.73 \\
\hline Cardiovascular & $205(49.4)$ & $360(61.4)$ & $<0.001$ \\
\hline Central nervous system & $142(34.2)$ & $241(41.1)$ & 0.03 \\
\hline Coagulation & $31(7.5)$ & $66(11.3)$ & 0.046 \\
\hline Hepatic & $15(3.6)$ & $37(6.3)$ & 0.06 \\
\hline
\end{tabular}


Table 1 Characteristics at baseline and on day one of intensive care unit admission. (Continued)

\begin{tabular}{lccc}
\hline Treatment on day one in the ICU & & $432(73.7)$ & $<0.001$ \\
Vasoactive agents & $247(59.5)$ & $469(80.0)$ & 0.12 \\
Mechanical ventilation & $315(75.9)$ & $51(8.7)$ & 0.91 \\
Renal replacement therapy & $37(8.9)$ & $143(24.4)$ & 0.81 \\
Glucocorticoids for septic shock & $104(25.1)$ &
\end{tabular}

${ }^{a}$ Culture-negative patients were more likely to have no comorbidities than culture-positive patients $\left(P=0.001\right.$, significant after Bonferroni correction); ${ }^{b}$ white blood cell values available for all patients; procalcitonin values available for 266 culture-negative and 359 culture-positive patients; C-reactive protein values available for 120 culture-negative patients and 174 culture-positive patients; ${ }^{C}$ organ failure defined as SOFA score $>2$ for the specified organ. ICU, intensive care unit; COPD, chronic obstructive pulmonary disease; APACHE, Acute Physiology and Chronic Health Evaluation; SOFA, Sequential Organ Failure Assessment.

Table 2 Site of infection.

\begin{tabular}{|c|c|c|c|}
\hline Site of infection ${ }^{a}$ & Culture-negative patients $(n=415)$ & Culture-positive patients $(n=586)$ & $P$ value \\
\hline Lungs & $309(74.5)$ & $351(59.9)$ & $<0.001$ \\
\hline Abdomen & $41(9.9)$ & $78(13.3)$ & 0.10 \\
\hline Enteritis and colitis & $22(5.3)$ & $18(3.1)$ & 0.08 \\
\hline Biliary & $8(1.9)$ & $25(4.3)$ & 0.04 \\
\hline Peritonitis & $6(1.4)$ & $13(2.2)$ & 0.38 \\
\hline Liver abscess & $1(0.2)$ & $17(2.9)$ & 0.002 \\
\hline Other abscess & $(0.2)$ & $4(0.7)$ & 0.41 \\
\hline Pancreatic & $1(0.2)$ & $1(0.2)$ & 1.00 \\
\hline Other abdominal & $2(0.5)$ & $0(0)$ & 0.17 \\
\hline Urinary tract & $17(4.1)$ & $71(12.1)$ & $<0.001$ \\
\hline Soft tissue and skin & $10(2.4)$ & $31(5.3)$ & 0.02 \\
\hline Nervous system & $2(0.5)$ & $8(1.4)$ & 0.21 \\
\hline Bones and joints & $2(0.5)$ & $8(1.4)$ & 0.21 \\
\hline Infective endocarditis & $2(0.5)$ & $14(2.4)$ & 0.02 \\
\hline Primary bacteremia & $0(0)$ & $12(2.0)$ & 0.002 \\
\hline Others & $14(3.4)$ & $15(2.6)$ & 0.45 \\
\hline Unknown & $18(4.3)$ & $29(4.9)$ & 0.65 \\
\hline
\end{tabular}

${ }^{\text {a }}$ Some patients had more than one site of infection.

Table 3 Cultures performed.

\begin{tabular}{|c|c|c|c|c|}
\hline Type of culture ${ }^{a}$ & Culture-negative patients $(n=415)$ & Culture-positive patients $(n=586)$ & $P$ value & Rate of culture positivity $(\%)^{\mathbf{b}}$ \\
\hline Blood & $410(98.8)$ & $579(98.8)$ & 1.00 & 20.3 \\
\hline Urine & $310(74.7)$ & $445(75.9)$ & 0.65 & 11.0 \\
\hline Endotracheal aspirate & $216(52.0)$ & $332(56.7)$ & 0.15 & 39.4 \\
\hline Expectorated sputum & $101(24.3)$ & $146(24.9)$ & 0.84 & 34.8 \\
\hline Bronchoalveolar lavage & $24(5.8)$ & $32(5.5)$ & 0.83 & 51.7 \\
\hline Pleural fluid & $12(2.9)$ & $14(2.4)$ & 0.62 & 14.8 \\
\hline Stool & $43(10.4)$ & $74(12.6)$ & 0.27 & 4.2 \\
\hline Peritoneal fluid & $9(2.2)$ & $22(3.8)$ & 0.15 & 20.6 \\
\hline Bile & $0(0)$ & $6(1.0)$ & 0.045 & 83.3 \\
\hline Liver abscess & $0(0)$ & $7(1.2)$ & 0.046 & 85.7 \\
\hline Other abscess & $1(0.2)$ & $3(0.5)$ & 0.65 & 60.0 \\
\hline Soft tissue and skin & $9(2.2)$ & $36(6.1)$ & 0.003 & 65.5 \\
\hline Cerebrospinal fluid & $8(1.9)$ & $8(1.4)$ & 0.48 & 12.5 \\
\hline Synovial fluid & $1(0.2)$ & $5(0.9)$ & 0.41 & 88.9 \\
\hline Others & $6(1.4)$ & $14(2.4)$ & 0.29 & 56.5 \\
\hline
\end{tabular}

${ }^{a}$ Cultures collected within two days before and the two days after ICU admission were included; ${ }^{\text {b}}$ calculated as (total number of positive cultures divided by total number of cultures performed) $\times 100$. ICU, intensive care unit. 
Table 4 Bacteria isolated.

\begin{tabular}{lcc}
\hline Bacteria isolated $^{\text {a }}$ & \multicolumn{2}{c}{ No. of patients (\% of entire cohort of patients) } \\
Bacteremia \\
\hline Gram-positive & $257(25.7)$ & $120(12.0)$ \\
Methicillin-sensitive Staphylococcus aureus & $82(8.2)$ & $39(39.0)$ \\
Methicillin-resistant Staphylococcus aureus & $59(5.9)$ & $10(1.0)$ \\
Streptococcus pneumoniae & $67(6.7)$ & $39(3.9)$ \\
Other Streptococcus species & $19(1.9)$ & $15(1.5)$ \\
Enterococcus & $28(2.8)$ & $16(1.6)$ \\
Others & $9(0.9)$ & $5(0.5)$ \\
Gram-negative & $390(39.0)$ & $213(21.3)$ \\
Klebsiella pneumoniae & $131(13.1)$ & $73(7.3)$ \\
Escherichia coli & $117(11.7)$ & $80(8.0)$ \\
Pseudomonas aeruginosa & $62(6.2)$ & $15(1.5)$ \\
Acinetobacter baumannii & $35(3.5)$ & $10(1.0)$ \\
Bulkholderia pseudomallei & $13(1.3)$ & $10(1.0)$ \\
Enterobacter cloacae & $10(1.0)$ & $4(0.4)$ \\
Hemophilus influenzae & $9(0.9)$ & $1(0.1)$ \\
Salmonella species & $7(0.7)$ & $3(0.3)$ \\
Citrobacter species & $6(0.6)$ & $3(0.3)$ \\
Stenotrophomonas maltophilia & $6(0.6)$ & $1(0.1)$ \\
Proteus species & $5(0.5)$ & $5(0.5)$ \\
Bacteroides fragilis & $5(0.5)$ & $2(0.2)$ \\
Others & $23(2.3)$ & $14(1.4)$ \\
\hline
\end{tabular}

${ }^{\mathrm{a}}$ More than one species of bacteria isolated in some patients.

Patient outcomes are presented in Table 5. Culturenegative patients had a shorter duration of hospital stay, and lower ICU mortality and hospital mortality (35.9\% versus $44.0 \%, P=0.01$ ) than culture-positive patients.

Table 6 details the variables associated with hospital mortality. While culture positivity was associated with higher mortality on univariable analysis, it did not feature as an independent predictor of mortality after accounting for other covariates on logistic regression analysis. The same applies to the administration of inappropriate antibiotics on the day of ICU admission. Multivariable analysis revealed the following independent predictors of mortality: age, time from hospitalization to ICU admission, lung, bone and joint infections, infective endocarditis, primary bacteremia, Acute Physiology Score, coagulation and hepatic failures, and mechanical ventilation on the day of ICU admission. The logistic regression model fitted well and there was no multicollinearity. In a separate analysis including specific microorganisms, Pseudomonas aeruginosa was the only pathogen which, when isolated independently, increased mortality (odds ratio (OR) 2.02, 95\% CI 1.08 to $3.79, P=0.03$ ).

Among the culture-positive patients, 265 were nonbacteremic while 321 were bacteremic. Hospital mortality was similar in both subgroups. The nonbacteremic but culture-positive subgroup had a higher mortality rate than the culture-negative group but this was not statistically significant; the bacteremic subgroup had a significantly higher mortality rate than the culture-negative group (Figure 1). Again among culture-positive patients, 467 received appropriate antibiotics on the first day of ICU stay while 119 did not. Hospital mortality was higher in the latter subgroup. The culture-positive subgroup that received appropriate antibiotics had a higher mortality rate than the culture-negative group but this was not statistically significant; the culture-positive subgroup that

Table 5 Outcomes.

\begin{tabular}{llll}
\hline & Culture-negative patients $(\boldsymbol{n}=\mathbf{4 1 5})$ & Culture-positive patients $(\boldsymbol{n}=\mathbf{5 8 6})$ & $\boldsymbol{P}$ value \\
\hline Hospital mortality & $149(35.9)$ & $258(44.0)$ & 0.01 \\
ICU mortality & $139(33.5)$ & $232(39.6)$ & 0.049 \\
Duration of mechanical ventilation, days ${ }^{\mathrm{a}}$ & $3.0(1.0-7.0)$ & $4.0(1.0-8.0)$ & 0.35 \\
Duration of ICU stay, days & $4.0(2.0-8.0)$ & $4.0(2.0-9.0)$ & 0.46 \\
Duration of hospital stay, days & $12.0(7.0-21.0)$ & $15.0(7.0-27.0)$ & 0.02 \\
\hline
\end{tabular}

${ }^{a} \mathrm{~A}$ total of 326 culture-negative patients and 448 culture-positive patients were mechanically ventilated during the ICU stay. ICU, intensive care unit. 
Table 6 Predictors of hospital mortality by univariable and multivariable logistic regression analyses.

\begin{tabular}{|c|c|c|c|c|}
\hline Variable $^{a}$ & Univariable OR $(95 \% \mathrm{Cl})$ & $P$ value & Multivariable adjusted OR $(95 \% \mathrm{Cl})^{\mathrm{b}}$ & $P$ value \\
\hline \multicolumn{5}{|l|}{ Demographics } \\
\hline Age, per year & $1.02(1.02-1.03)$ & $<0.001$ & $1.03(1.02-1.04)$ & $<0.001$ \\
\hline \multicolumn{5}{|l|}{ Source of admission } \\
\hline Hospital floor versus ED & $1.90(1.46-2.48)$ & $<0.001$ & $1.34(0.97-1.85)$ & 0.80 \\
\hline Other ICU versus ED & $1.38(1.05-1.81)$ & 0.02 & $1.24(0.64-2.38)$ & 0.53 \\
\hline \multicolumn{5}{|l|}{ Time of ICU admission } \\
\hline Per day from hospitalization & $1.06(1.03-1.09)$ & $<0.001$ & $1.06(1.03-1.09)$ & $<0.001$ \\
\hline \multicolumn{5}{|l|}{ Comorbid conditions } \\
\hline Chronic kidney disease & $1.51(1.06-2.15)$ & 0.02 & $1.29(0.80-2.08)$ & 0.29 \\
\hline Hematological malignancy & $2.03(1.01-4.09)$ & 0.048 & $1.40(0.55-3.57)$ & 0.48 \\
\hline \multicolumn{5}{|l|}{ Site of infection } \\
\hline Lungs & $0.98(0.75-1.27)$ & 0.85 & $2.04(1.00-4.16)$ & 0.049 \\
\hline Bones and joints & $5.94(1.25-28.09)$ & 0.03 & $7.31(1.05-50.77)$ & 0.04 \\
\hline Infective endocarditis & $1.90(0.70-5.13)$ & 0.21 & $5.43(1.41-20.87)$ & 0.01 \\
\hline Primary bacteremia & $4.46(1.20-16.56)$ & 0.03 & $8.31(1.55-44.49)$ & 0.01 \\
\hline \multicolumn{5}{|l|}{ Microbiology and antimicrobials } \\
\hline Culture-positive & $1.40(1.08-1.82)$ & 0.01 & $0.88(0.59-1.31)$ & 0.52 \\
\hline Inappropriate antibiotics & $1.42(1.17-1.71)$ & $<0.001$ & $0.86(0.52-1.42)$ & 0.56 \\
\hline \multicolumn{5}{|l|}{ Severity scores } \\
\hline APS, per point & $1.07(1.05-1.09)$ & 0.001 & $1.06(1.03-1.09)$ & $<0.001$ \\
\hline \multicolumn{5}{|l|}{ Organ failure ${ }^{c}$} \\
\hline Renal & $1.36(1.00-1.84)$ & 0.049 & $0.99(0.64-1.55)$ & 0.98 \\
\hline Respiratory & $1.31(1.02-1.70)$ & 0.04 & $0.98(0.70-1.36)$ & 0.90 \\
\hline Cardiovascular & $1.71(1.32-2.21)$ & $<0.001$ & $0.91(0.57-1.44)$ & 0.68 \\
\hline Central nervous system & $2.17(1.67-2.82)$ & $<0.001$ & $1.15(0.80-1.65)$ & 0.45 \\
\hline Coagulation & $3.02(1.95-4.68)$ & $<0.001$ & $2.59(1.51-4.46)$ & 0.001 \\
\hline Hepatic & $4.27(2.28-7.98)$ & $<0.001$ & $6.30(2.98-13.30)$ & $<0.001$ \\
\hline \multicolumn{5}{|l|}{ Treatment on day 1 in the ICU } \\
\hline Vasoactive agents & $2.07(1.56-2.76)$ & $<0.001$ & $1.42(0.88-2.30)$ & 0.15 \\
\hline Mechanical ventilation & $3.17(2.23-4.52)$ & $<0.001$ & $1.91(1.21-3.02)$ & 0.005 \\
\hline
\end{tabular}

${ }^{a}$ Only variables that were significantly associated with hospital mortality on univariable or multivariable analyses are shown; ${ }^{\mathrm{b}} \mathrm{Hosmer-Lemeshow} \mathrm{test} \mathrm{for}$ goodness of fit for multivariable logistic regression model: $\chi^{2}=7.208$, degrees of freedom $=8, P=0.51$; ' organ failure defined as Sequential Organ Failure Assessment (SOFA) score $>2$ for the specified organ. OR, odds ratio; Cl, confidence interval; ED, emergency department; ICU, intensive care unit; APS, Acute Physiology Score, used in place of the Acute Physiology and Chronic Health Evaluation (APACHE) II score.

did not receive appropriate antibiotics had a significantly higher mortality rate than the culture-negative group (Figure 2).

\section{Discussion}

To the best of our knowledge, no previous study has focused on the differences between culture-negative and culture-positive severe sepsis. The main findings of this study are that patients with culture-negative sepsis had fewer comorbidities and lower severity of illness than those with culture-positive sepsis. Although culturenegative patients had a shorter hospitalization and lower ICU mortality and hospital mortality than culture-positive patients, culture positivity per se was not independently associated with mortality on multivariable analysis.

Causative microorganisms were not found in $41.5 \%$ of our patients. Multicenter studies published in the last decade found that culture-negative patients accounted for $28 \%, 35 \%, 38 \%$, and $48 \%$ of all cases of severe sepsis in North American, Spanish, French, and Canadian ICUs, respectively [10,16-18]. The corresponding figure was $40 \%$ in the pan-European Sepsis Occurrence in Acutely Ill Patients (SOAP) study [11]. In the United States, $49 \%$ of hospitalized patients with sepsis were culture-negative [3]. Meanwhile, the Extended Prevalence of Infection in Intensive Care (EPIC) II study, which reported prevalence - and not incidence - found that $30 \%$ of all infections in ICUs worldwide were culturenegative [19]. While we found more Gram-negative pathogens, others have found a predominance of Grampositive microorganisms [3,11].

Despite the high prevalence of culture-negative sepsis, studies which focus on the outcomes of such patients are surprisingly limited. In the 1990s, Rangel-Frausto 


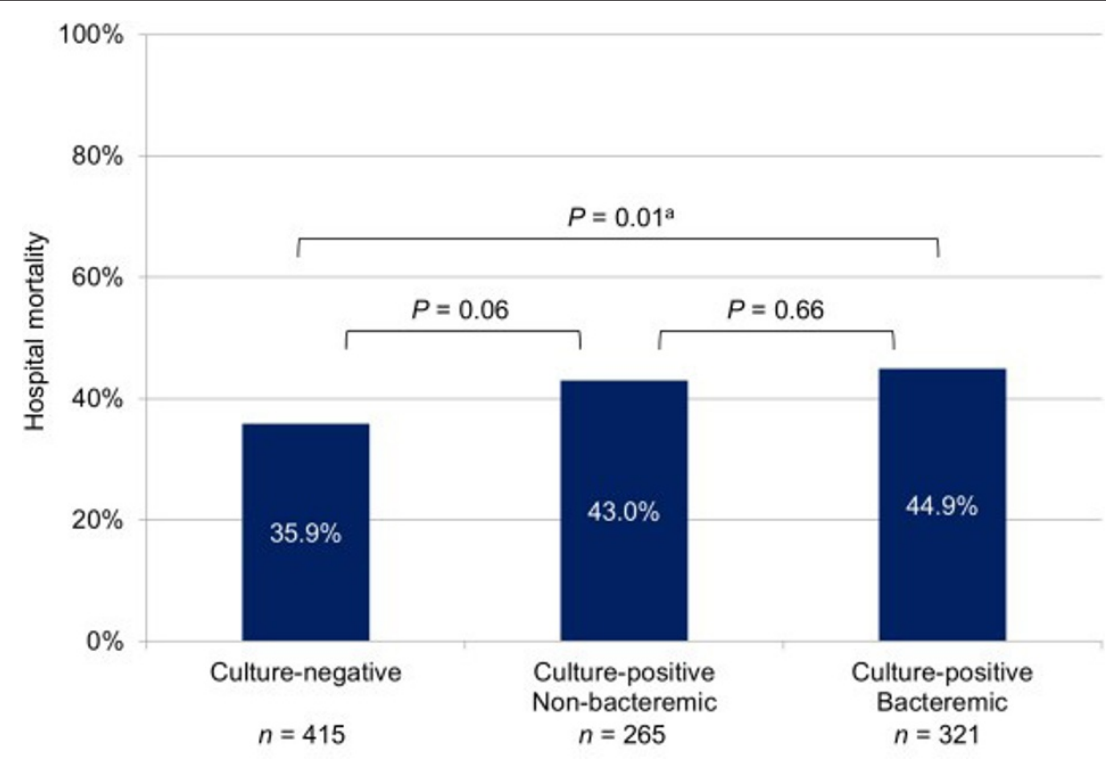

Figure 1 Hospital mortality for subgroups according to cultures and bacteremia. Overall $P$ value for comparison between three subgroups

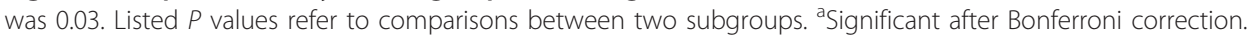

and colleagues found a mortality of $25 \%$ among 577 patients with severe sepsis and septic shock in a teaching hospital and no difference in outcomes between culturenegative and culture-positive patients [9]. Mortality was also similar among 310 culture-negative and 742 culturepositive patients with severe sepsis in a multicenter study by Brun-Buisson and colleagues [8]. In the 2000s, a North American study led by Kumar and colleagues found similar mortality among 608 culture-negative and
1,546 culture-negative patients [10], while the panEuropean SOAP study found similar ICU mortality rates (40\% versus 39\%) between 468 culture-negative and 454 culture-positive septic patients [11]. Our study, on the other hand, found a significantly lower hospital mortality rate for culture-negative than for culture-positive severe sepsis (35.9\% versus $44.0 \%)$. Although this difference was greater for bacteremic patients than for culture-positive but nonbacteremic patients, there was no significant

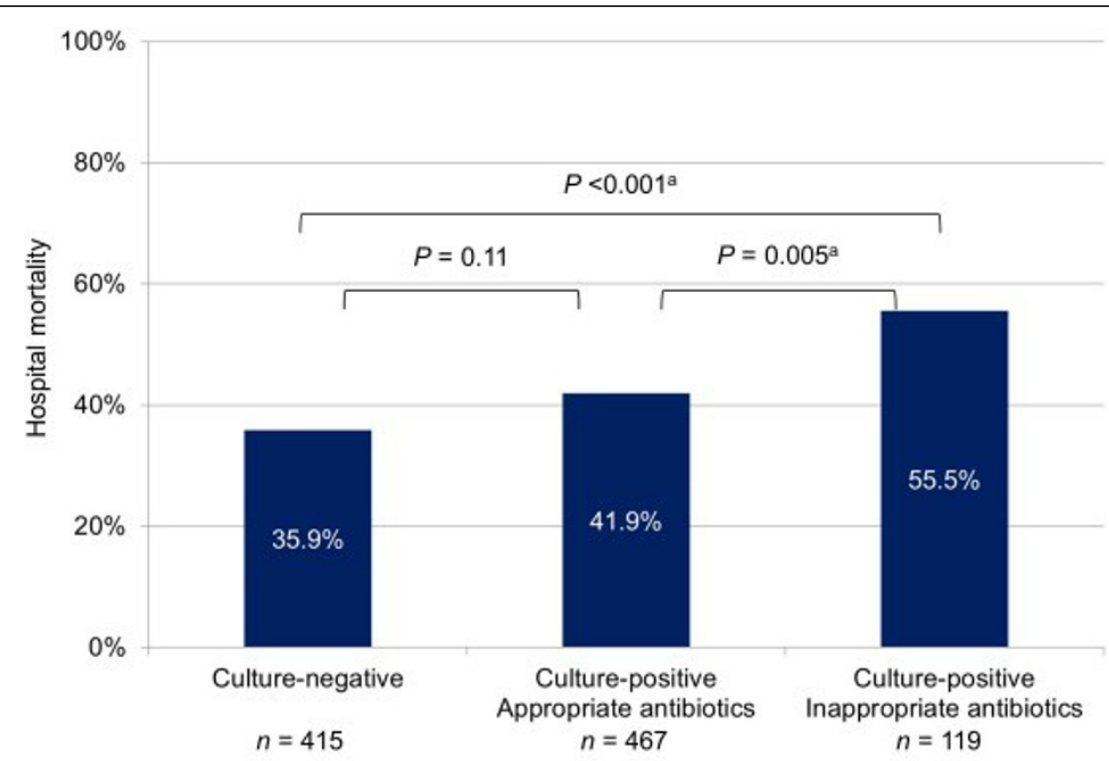

Figure 2 Hospital mortality for subgroups according to cultures and receipt of appropriate antibiotics. Overall $P$ value for comparison between three subgroups was 0.005 . Listed $P$ values refer to comparisons between two subgroups. ${ }^{a}$ Significant after Bonferroni correction. 
difference in mortality between these latter two subgroups $(43.0 \%$ versus $44.9 \%)$. The reasons why we found a difference in mortality when the earlier studies did not are not immediately clear. These studies were not primarily designed for such a comparison and did not provide details on the severity of illness in these two groups of patients. In Rangel-Frausto et al.'s cohort, culture-negative patients were less likely to have acute kidney injury and shock, but in Brun-Buisson et al.'s cohort, culture-negative patients had more hypotension [8,9]. In our study, culturenegative patients were clearly less sick than their culturepositive counterparts: they had fewer comorbidities, less hemodynamic instability and organ failures, and lower APACHE II scores. After adjustment for covariates including severity of illness, identification of microorganisms was not independently associated with mortality, a finding similarly reported recently by the French OUTCOMEREA database [12].

The question that then begs to be answered is: what exactly is the cause of culture-negative sepsis? Is it merely a milder form of sepsis compared to culture-positive sepsis? While our study cannot answer these questions, its findings provide some insight into four possibilities. First, it is known that cultures lack the sensitivity to identify all bacteria. Postulated reasons include prior antibiotic exposure, sampling error, insufficient volume for blood cultures, poor transport conditions, and slow-growing or fastidious bacteria [6]. Polymerase chain reaction (PCR)based molecular techniques may improve detection rates, and many patients with clinical sepsis are indeed PCRpositive but culture-negative [20-22]. In the PIRO system for staging sepsis, the letter 'I' refers to the nature and extent of the infection [23]. It may be hypothesized that the lower severity of culture-negative sepsis in our study was at least in part due a milder insult and lower bacterial burden [24], and correspondingly, the inability to capture the microorganisms on cultures. While it is possible that antibiotic pretreatment might have contributed to negative cultures, this is less likely for blood cultures that were usually performed before antimicrobial therapy. In addition, although we did not differentiate communityacquired from hospital-acquired infections, the short median (interquartile range (IQR)) lag time from presentation to the hospital to ICU admission of 0 days (0 to 2$)$ suggests that most patients had the former, where the incidence of antibiotic pretreatment is likely to be lower. The letter 'P' in the PIRO system refers to predisposition [23], and given the trend toward more culture-positive sepsis among diabetic patients in our cohort, it is conceivable that diabetics are more prone to having large bacterial loads [25].

Second, it is clear from our data that certain infections are less common in culture-negative than in culture- positive patients, and vice versa. This is in part due to the nature of some infections, for example, liver abscesses are less likely to be culture-negative [26], and in part due to the diagnostic criteria for other infections, for example, the importance of blood culture positivity for primary bacteremia and in the revised Duke criteria for infective endocarditis.

Third, some of our culture-negative patients might have had nonbacterial sepsis. Fungi account for approximately $5 \%$ of cases of sepsis in ICUs and are generally more readily detected than viruses and parasites $[16,17]$. Our study design mandated the exclusion of such microorganisms. While we are confident that most patients with fungal sepsis were diagnosed and thus excluded, and that parasites are extremely rare in our urban setting, it is plausible that undetected viruses contributed to a significant proportion of culture-negative sepsis. Using reverse-transcription PCR assays on bronchoalveolar lavage fluid and nasopharyngeal swab specimens, Choi and colleagues recently demonstrated that severe pneumonia was due to viruses $36 \%$ of the time [27]. It is not routine clinical practice in most ICUs, including ours, to test for viruses in pneumonia, and the lungs were a commoner site of infection in culturenegative than in culture-positive patients in our study. Meanwhile, serum procalcitonin levels, which are a marker of bacterial as opposed to viral infections, were lower in our culture-negative group, again bringing up the possibility that viruses played a role [28].

Importantly, a fourth conceivable explanation for our culture-negative group is that some of the patients did not actually have sepsis. The 1992 ACCP/SCCM Consensus Conference criteria that we and many other investigators used to define sepsis $[11,17,18]$ - as well as subsequent adaptations - are based on a composite of clinical and laboratory data and will inevitably include a heterogeneous set of diagnoses, some of which are false positives and unrelated to infections $[13,14,23]$. In a study by Heffner and colleagues, $32 \%$ of culture-negative patients who were initially identified as having severe sepsis in the emergency department were subsequently found to have noninfectious mimics while $16 \%$ had illnesses of indeterminate etiology [29]. Among our culture-negative patients, $74.5 \%$ had a lung infection, compared to $64 \%$ in the European SOAP cohort [11]. This might have been due to the fact that ours is a medical ICU, but one could also postulate that some of our patients had mimickers of pneumonia such as heart failure. However, because we used all available clinical information from ICU admission till discharge or death before labeling severe sepsis, including noninvasive and invasive hemodynamic monitoring where indicated, it is likely that the proportion of patients who did not actually 
have sepsis in our study was smaller than in Heffner and colleagues' cohort.

What are the implications of our study's findings? Early administration of broad-spectrum antibiotics is recommended by the Surviving Sepsis Campaign guidelines in an effort to improve outcomes in sepsis, culturepositive or culture-negative [5]. Kumar and colleagues showed that every hour of delay in the administration of effective antibiotics from the onset of septic shock resulted in an increase in mortality [10]. This association occurred even among culture- negative patients, for which antimicrobial therapy was deemed appropriate if they were consistent with national guidelines modified to local flora for the clinical syndrome. In contrast, similar to the majority of studies in the literature [4], our study defined the administered antibiotics to be inappropriate only if they did not match the in vitro susceptibility of the identified pathogens, that is, only in culture-positive patients. In so doing, we found the mortality rate to be much higher among culture-positive patients who were administered inappropriate antibiotics than culturenegative patients (55.5\% versus $35.9 \%)$. There was also a trend toward higher mortality among culture-positive patients who received appropriate antibiotics (41.9\%) than culture-negative patients. These findings do not invalidate the Surviving Sepsis Campaign's recommendation, especially since it is impossible to accurately predict one's culture status at presentation. On the clinical front, we echo the Surviving Sepsis Campaign guidelines' advice for cautious consideration of antimicrobial therapy using clinician judgment and available clinical information when cultures are unrevealing [5]. On the research front, we propose that it is time for more in-depth studies of culture-negative sepsis. Such investigations could come in the form of multiplex PCR amplification techniques for the quantification of bacteria, fungi, and viruses to elucidate the false-negative and true-negative rates of cultures $[20,24]$, and interventional trials comparing algorithms to escalate, continue, narrow, or cease antibiotics coupled with a search for noninfectious etiologies when pathogens are not detected [30].

Our study has several limitations. First, it compartmentalizes sepsis into two main groups based on the identification, or lack thereof, of pathogenic microorganisms, but in reality, both groups are a mixed bag of diagnoses [31]. As discussed at length above, the culture-negative group probably included some patients with nonbacterial sepsis and patients without sepsis. Nonetheless, the incidence of culture-negative sepsis in our cohort mirrors that in multiple studies internationally $[3,10,11,16-18]$, and this reinforces the need to better understand this real-world phenomenon. As for the culture-positive group, Pseudomonas aeruginosa was the only bacteria that independently increased mortality, a finding that has also been seen in European ICUs [11]. Besides, bacteremia is often seen as a harbinger of bad outcomes and thus may not be equivalent to the identification of pathogens in bodily fluids other than blood [31,32]. This notwithstanding, our analyses suggest no difference in mortality between bacteremic and nonbacteremic culture-positive sepsis. Second, because we left the performance of cultures to the discretion of the managing physicians, we are unable to rule out the fact that some patients were culture-negative simply because of inappropriate sampling. Nonetheless, given that most cultures were performed in an equal proportion of patients in the culture-negative and the culture-positive groups, this is less likely to be a significant contributing factor to our findings. Third, as ours was a single-center study conducted in a medical ICU, the details of its findings may not be extrapolated to all ICU patients. Fourth, to determine the appropriateness of antimicrobial therapy, we did not record the exact timing of administration while reviewing the antibiotics given on the first day of ICU stay. This timeline is longer than the three-hour window period from the onset of sepsis suggested by the Surviving Sepsis Campaign [5]. Nonetheless, we found in a previous study that antibiotics were administered within three hours $75.0 \%$ of the time at our ICU [33]. Fifth, as our study was observational in nature, unadjusted and hidden confounders might have influenced our results and conclusions. To illustrate, although it is unlikely that significant differences exist in the acute management of the culturenegative and the culture-positive groups, especially when microbiological results would not have been available at presentation, it should still be acknowledged that data on treatments such as fluid resuscitation are lacking. Our study also has several strengths. It is the first and largest prospective epidemiological study dedicated to the difference between culture-negative and culture-positive severe sepsis. We only included microorganisms that were deemed by the managing physicians to be pathogens as opposed to colonizers or contaminants, with reference to the International Sepsis Forum Consensus Conference guidelines [14]. To optimize accuracy, data checks were performed by the investigators.

\section{Conclusions}

Our study identified significant differences between culture-negative and culture-positive severe sepsis, with the former group having fewer comorbidities, milder severity of illness, shorter hospitalizations, and lower ICU mortality and hospital mortality. However, after adjusting for all covariates, culture positivity did not independently predict mortality. 


\section{Key messages}

- A large proportion of patients with severe sepsis are culture-negative.

- Culture-negative patients have fewer comorbidities and lower severity of illness than culture-positive patients.

- Culture-negative patients have a shorter hospitalization than culture-positive patients.

- Although culture-negative patients have lower ICU mortality and hospital mortality than culture-positive patients, culture positivity per se is not independently associated with mortality on multivariable analysis.

- More research is required to better understand the underlying causes of culture-negative severe sepsis.

\section{Abbreviations}

ACCP: American College of Chest Physicians; APACHE: Acute Physiology and Chronic Health Evaluation; Cl: confidence interval; EPIC: Extended Prevalence of Infection in Intensive Care; ICU: intensive care unit; PCR: polymerase chain reaction; SCCM: Society of Critical Care Medicine; SOAP: Sepsis Occurrence in Acutely III Patients; SOFA: Sequential Organ Failure Assessment.

Competing interests

The authors declare that they have no competing interests.

\section{Authors' contributions}

$J P, W J N$, and KHL conceived the study and participated in its design and coordination. JP, WJN, KCS, CKT, TK, HFL, MYC, HSY, AT, HJK, RC, KHL, and AM participated in the data analysis and interpretation. JP drafted the manuscript. JP, WJN, KCS, CKT, TK, HFL, MYC, HSY, AT, HJK, RC, KHL, and AM participated in the revision of the manuscript, and read and approved the final manuscript.
\end{abstract}

\section{Acknowledgements}

We would like to sincerely thank Ting Yanghan Yohanes, Ramana Narendran, Tan Wei Loong Barry, and Tan Chin Hung Barry for the assistance provided during the study. This study was unfunded.

\section{Authors' details}

'Division of Respiratory and Critical Care Medicine, University Medicine Cluster, National University Hospital, National University Health System Tower Block Level 10, 1E Kent Ridge Road, Singapore 119228. ${ }^{2}$ Department of Medicine, Yong Loo Lin School of Medicine, National University of Singapore, National University Health System Tower Block Level 10, 1E Kent Ridge Road, Singapore 119228. ${ }^{3}$ Faculty of Medicine, Suite H 2743, 300 Prince Philip Drive, Memorial University of Newfoundland, St. John's NL, A1B 3V6, Canada. ${ }^{4}$ Department of Medicine, Alexandra Hospital (Jurong Health Services), 378 Alexandra Road, Singapore 159964. ${ }^{5}$ RIPAS Hospital, Jalan Putera Al-Muhtadee Billah, Bandar Seri Begawan, BA 1710, Brunei Darussalam. ${ }^{6}$ Asian Centre for Liver Diseases and Transplantation, Gleneagles Hospital, Annexe Block, 02-37, 6A Napier Road, Singapore 258500.

Received: 31 January 2013 Revised: 30 April 2013

Accepted: 12 September 2013 Published: 12 September 2013

\section{References}

1. Russell JA: Management of sepsis. N Engl J Med 2006, 355:1699-1713.

2. Annane $D$, Aegerter $P$, Jars-Guincestre MC, Guidet B: Current epidemiology of septic shock: the CUB-Rea Network. Am J Respir Crit Care Med 2003, 168:165-172.

3. Martin GS, Mannino DM, Eaton S, Moss M: The epidemiology of sepsis in the United States from 1979 through 2000. N Engl J Med 2003, 348:1546-1554.

4. Paul M, Shani V, Muchtar E, Kariv G, Robenshtok E, Leibovici L: Systematic review and meta-analysis of the efficacy of appropriate empiric antibiotic therapy for sepsis. Antimicrob Agents Chemother 2010, 54:4851-4863.

5. Dellinger RP, Levy MM, Rhodes A, Annane D, Gerlach H, Opal SM, Sevransky JE, Sprung CL, Douglas IS, Jaeschke R, Osborn TM, Nunnally ME, Townsend SR, Reinhart K, Kleinpell RM, Angus DC, Deutschman CS, Machado FR, Rubenfeld GD, Webb SA, Beale RJ, Vincent JL, Moreno R: Surviving Sepsis Campaign: international guidelines for management of severe sepsis and septic shock: 2012. Crit Care Med 2013, 41:580-637.

6. Lever A, Mackenzie I: Sepsis: definition, epidemiology, and diagnosis. BMJ 2007, 335:879-883

7. Alberti C, Brun-Buisson C, Burchardi H, Martin C, Goodman S, Artigas A, Sicignano A, Palazzo M, Moreno R, Boulme R, Lepage E, Le Gall R: Epidemiology of sepsis and infection in ICU patients from an international multicentre cohort study. Intensive Care Med 2002, 28:108-121.

8. Brun-Buisson C, Doyon F, Carlet J, Dellamonica P, Gouin F, Lepoutre A, Mercier JC, Offenstadt G, Regnier B: Incidence, risk factors, and outcome of severe sepsis and septic shock in adults. A multicenter prospective study in intensive care units. French ICU Group for Severe Sepsis. JAMA 1995, 274:968-974

9. Rangel-Frausto MS, Pittet D, Costigan M, Hwang T, Davis CS, Wenzel RP: The natural history of the systemic inflammatory response syndrome (SIRS). A prospective study. JAMA 1995, 273:117-123.

10. Kumar A, Roberts D, Wood KE, Light B, Parrillo JE, Sharma S, Suppes R, Feinstein D, Zanotti S, Taiberg L, Gurka D, Kumar A, Cheang M: Duration of hypotension before initiation of effective antimicrobial therapy is the critical determinant of survival in human septic shock. Crit Care Med 2006, 34:1589-1596.

11. Vincent IL, Sakr Y, Sprung CL, Ranieri VM, Reinhart K, Gerlach H, Moreno R, Carlet J, Le Gall JR, Payen D: Sepsis in European intensive care units: results of the SOAP study. Crit Care Med 2006, 34:344-353.

12. Zahar JR, Timsit JF, Garrouste-Orgeas M, Francais A, Vesin A, DescorpsDeclere A, Dubois Y, Souweine B, Haouache H, Goldgran-Toledano D, Allaouchiche B, Azoulay E, Adrie C: Outcomes in severe sepsis and patients with septic shock: pathogen species and infection sites are not associated with mortality. Crit Care Med 2011, 39:1886-1895.

13. American College of Chest Physicians/Society of Critical Care Medicine Consensus Conference: definitions for sepsis and organ failure and guidelines for the use of innovative therapies in sepsis. Crit Care Med 1992, 20:864-874.

14. Calandra T, Cohen J: The international sepsis forum consensus conference on definitions of infection in the intensive care unit. Crit Care Med 2005, 33:1538-1548.

15. Dellinger RP, Carlet JM, Masur H, Gerlach H, Calandra T, Cohen J, GeaBanacloche J, Keh D, Marshall JC, Parker MM, Ramsay G, Zimmerman JL, Vincent JL, Levy MM: Surviving Sepsis Campaign guidelines for management of severe sepsis and septic shock. Crit Care Med 2004, 32:858-873.

16. Blanco J, Muriel-Bombin A, Sagredo V, Taboada F, Gandia F, Tamayo L, Collado J, Garcia-Labattut A, Carriedo D, Valledor M, De Frutos M, Lopez MJ, Caballero A, Guerra J, Alvarez B, Mayo A, Villar J: Incidence, organ dysfunction and mortality in severe sepsis: a Spanish multicentre study. Crit Care 2008, 12:R158.

17. Brun-Buisson C, Meshaka P, Pinton P, Vallet B: EPISEPSIS: a reappraisal of the epidemiology and outcome of severe sepsis in French intensive care units. Intensive Care Med 2004, 30:580-588.

18. Martin CM, Priestap F, Fisher H, Fowler RA, Heyland DK, Keenan SP, Longo CJ, Morrison T, Bentley D, Antman N: A prospective, observational registry of patients with severe sepsis: the Canadian Sepsis Treatment and Response Registry. Crit Care Med 2009, 37:81-88.

19. Vincent JL, Rello J, Marshall J, Silva E, Anzueto A, Martin CD, Moreno R, Lipman J, Gomersall C, Sakr Y, Reinhart K: International study of the prevalence and outcomes of infection in intensive care units. JAMA 2009, 302:2323-2329.

20. Dark PM, Dean P, Warhurst G: Bench-to-bedside review: the promise of rapid infection diagnosis during sepsis using polymerase chain reactionbased pathogen detection. Crit Care 2009, 13:217.

21. Bloos F, Hinder F, Becker K, Sachse S, Mekontso Dessap A, Straube E, Cattoir V, Brun-Buisson C, Reinhart K, Peters G, Bauer M: A multicenter trial to compare blood culture with polymerase chain reaction in severe human sepsis. Intensive Care Med 2010, 36:241-247. 
22. Louie RF, Tang Z, Albertson TE, Cohen S, Tran NK, Kost GJ: Multiplex polymerase chain reaction detection enhancement of bacteremia and fungemia. Crit Care Med 2008, 36:1487-1492.

23. Levy MM, Fink MP, Marshall JC, Abraham E, Angus D, Cook D, Cohen J, Opal SM, Vincent JL, Ramsay G: 2001 SCCM/ESICM/ACCP/ATS/SIS International Sepsis Definitions Conference. Crit Care Med 2003, 31:1250-1256.

24. Lisboa T, Waterer G, Rello J: We should be measuring genomic bacterial load and virulence factors. Crit Care Med 2010, 38:S656-662.

25. Thomsen RW, Hundborg HH, Lervang HH, Johnsen SP, Schonheyder HC, Sorensen HT: Diabetes mellitus as a risk and prognostic factor for community-acquired bacteremia due to enterobacteria: a 10-year, population-based study among adults. Clin Infect Dis 2005, 40:628-631.

26. Tsai FC, Huang YT, Chang LY, Wang JT: Pyogenic liver abscess as endemic disease, Taiwan. Emerg Infect Dis 2008, 14:1592-1600.

27. Choi SH, Hong SB, Ko GB, Lee Y, Park HJ, Park SY, Moon SM, Cho OH, Park KH, Chong YP, Kim SH, Huh JW, Sung H, Do KH, Lee SO, Kim MN, Jeong JY, Lim CM, Kim YS, Woo JH, Koh Y: Viral infection in patients with severe pneumonia requiring intensive care unit admission. Am J Respir Crit Care Med 2012, 186:325-332.

28. Schuetz P, Chiappa V, Briel M, Greenwald JL: Procalcitonin algorithms for antibiotic therapy decisions: a systematic review of randomized controlled trials and recommendations for clinical algorithms. Arch Intern Med 2011, 171:1322-1331.

29. Heffner AC, Horton JM, Marchick MR, Jones AE: Etiology of illness in patients with severe sepsis admitted to the hospital from the emergency department. Clin Infect Dis 2010, 50:814-820.

30. Heenen $S$, Jacobs F, Vincent JL: Antibiotic strategies in severe nosocomial sepsis: why do we not de-escalate more often? Crit Care Med 2012, 40:1404-1409.

31. Cohen J, Cristofaro P, Carlet J, Opal S: New method of classifying infections in critically ill patients. Crit Care Med 2004, 32:1510-1526.

32. Hugonnet S, Harbarth S, Ferriere K, Ricou B, Suter P, Pittet D: Bacteremic sepsis in intensive care: temporal trends in incidence, organ dysfunction, and prognosis. Crit Care Med 2003, 31:390-394.

33. Phua J, Ho BC, Tee A, Chan KP, Johan A, Loo S, So CR, Chia N, Tan AY, Tham HM, Chan YH, Koh Y: The impact of clinical protocols in the management of severe sepsis: a prospective cohort study. Anaesth Intensive Care 2012, 40:663-674.

doi:10.1186/cc12896

Cite this article as: Phua et al:: Characteristics and outcomes of culturenegative versus culture-positive severe sepsis. Critical Care 2013 17:R202.

\section{Submit your next manuscript to BioMed Central and take full advantage of:}

- Convenient online submission

- Thorough peer review

- No space constraints or color figure charges

- Immediate publication on acceptance

- Inclusion in PubMed, CAS, Scopus and Google Scholar

- Research which is freely available for redistribution

Submit your manuscript at www.biomedcentral.com/submit 